Exotic Stars as Challenges to Evolution

ASP Conference Series, Vol. 279, 2002

C. A. Tout and W. Van Hamme, eds.

\title{
Fluorescence Model for AG Draconis
}

\author{
W. Van Hamme \\ Department of Physics, Florida International University, Miami, FL \\ 33199, USA
}

R. E. Wilson

Astronomy Department, University of Florida, Gainesville, FL 32611,
USA

\begin{abstract}
A fluorescent light curve model (ionization-bounded wind and chromosphere with scale height) that previously found most fluorescent variation in symbiotic nova V1329 Cygni to be chromospheric, is applied to AG Draconis, where the strong wind of a well-detached red giant is illuminated by ultraviolet radiation from an accreting white dwarf. Bandpass dependence of light curve amplitude is even more striking than in V1329 Cyg and eliminates thermal re-radiation from the red star photosphere as a principal variation mechanism, since thermal re-radiation cannot change by large factors from $U$ to $B$ to $V$. Spectra show strong continuum and line emission, so both light curves and spectra show the importance of fluorescence. A small secular decay was included by fitting in time rather than phase, with $d T_{\mathrm{wd}} / d t$ as a parameter. There is a $3 \sigma$ indication of a large negative $d P / d t$. Chromospheric fluorescence accounts for the entire $U$ variation, while $B$ and $V$ variations are too small . for wind and chromosphere to be distinguished. We confirm a previously identified 355-day radial velocity period that is consistent with red star pulsation.
\end{abstract}

\section{Introduction}

Many symbiotic binaries, and especially symbiotic novae, show fluorescence as a small hot star illuminates a red giant wind and/or chromosphere. The fluorescent region may be difficult to locate from spectra because spectral variations of winds and chromospheres can be similar and because large numbers of spectra that are well distributed in time seldom exist. Radial velocities may be too inaccurate to help because orbital motions are slow. How can one identify fluorescent regions against this backdrop of difficulties? A fluorescent light curve model (Wilson 1999; 2001) can identify regions of bright fluorescence and distinguish wind from chromospheric fluorescence. The model has a constant speed wind with an ionization front, as in Seaquist, Taylor, \& Button $(1984 ; 1987)$ and a chromosphere with a scale height, both fluorescing under ultraviolet radiation from a small hot star. Far UV radiation is converted to optical light with bandpass conversion efficiencies. The idea is to track combined emission line 
flux in photometric bands rather than individual lines. There are versions I and II of the model (viz. Chochol \& Wilson 2001), with Model I applied here. Model II may be tried later.

Note that the problem has major radiative and geometrical parts and it is important to have a model that interfaces effectively with existing observations. With the model, Chochol \& Wilson (2001) found that most fluorescent variation in symbiotic nova V1329 Cygni is chromospheric, although the wind is brighter than the chromosphere in $B$ and $V$ and is similar to the time-averaged chromospheric brightness in $U$. The fluorescent wind/chromosphere light curve model may be a useful diagnostic for other binaries. Indeed the well studied symbiotic star AG Draconis shows a wave-like variation on the orbit period $(\approx 0.3 \mathrm{semi}$ amplitude in $U$ and much smaller in $B, V$ ) that appears to originate much as in V1329 Cyg, so we looked into whether the fluorescent light curve model can realistically probe AG Dra.

\section{Modeling AG Draconis}

AG Dra is a high velocity $\left(-146 \mathrm{~km} \mathrm{~s}^{-1}\right)$ symbiotic nova with orbital period 554 days and $a \approx 1.5 \mathrm{AU}$. When not in outburst, the binary has $U \approx 11^{\mathrm{m}} 2$, $B \approx 11^{\mathrm{m}} 1$, and $V \approx 9.7$. The currently accepted configuration is that of a KIII giant with a strong wind, illuminated by ultraviolet radiation from the vicinity of a hot accreting white dwarf, perhaps with $T_{\mathrm{wd}} \approx 10^{5} \mathrm{~K}$ and with the giant comfortably detached. The orbital inclination is uncertain, but no star-star eclipses are apparent. Distance estimates vary widely, although $2 \mathrm{kpc}$ should be of the right order. Ordinary thermal re-emission (reflection) has been advocated as the cause of the optical wave (Formiggini \& Leibowitz 1990) and the form of variation does resemble that of the reflection effect, but light curves and spectra independently show that reflection does not cause the wave. Thermal radiation cannot change by large factors from $U$ to $B$ to $V$, as seen in AG Dra light curves. Optical spectra (e.g. Mikołajewska et al. 1995; Huang, Chen, \& Chen 1988; Munari \& Zwitter 2002) demonstrate that the spectral regions most dominated by emission lines and Balmer continuum emission are those that show the wave in broad band photometry. A phenomenon geometrically similar to thermal re-emission but radiatively far different is chromospheric fluorescence due to irradiance, and our light curve modeling supports it as the mechanism of AG Dra's wave.

The interval JD 2443900 to 2452000 is marked by several nova-like outbursts of order $3 \mathrm{mag}$ in $U$ that we avoided, as our interest is in the emission in quiescence that varies on the orbit period and thereby provides information on the geometry of fluorescent regions via eclipses and possible eccentricityrelated variations. We removed the outbursts, leaving quiescent intervals with their wave-like behavior superimposed on a slow secular decay, and carried out solutions in time rather than phase so that the decay could be parameterized. Specifically we solved for $d T_{\mathrm{wd}} / d t$, where $T_{\mathrm{wd}}$ is the temperature of the hot object, and for $d P / d t$ ( $P$ is orbital period). Our experience over the past 6 years has shown that $d P / d t$ can sometimes be found more accurately from whole light and velocity curves than from the traditional method of eclipse minima (Elias et al. 1997; Van Hamme \& Wilson 1998; Van Hamme et al. 2001; Chochol \& Wilson 
2001). In this case the velocity curves alone did not lead to a measurable $d P / d t$ (Table 1) but a combined light-velocity solution found a very large negative $d P / d t$ at the $3 \sigma$ level (Table 2). Parameter definitions are in Chochol \& Wilson (2001). While the decay time scale of approximately $10^{3}$ years seems alarmingly short, it may characterize only a brief transient stage and, although the nonzero $d P / d t$ is unlikely to be real, it may be too early to dismiss this purely observational result. Dimensions and other parameters are in Tables 1 and 2.

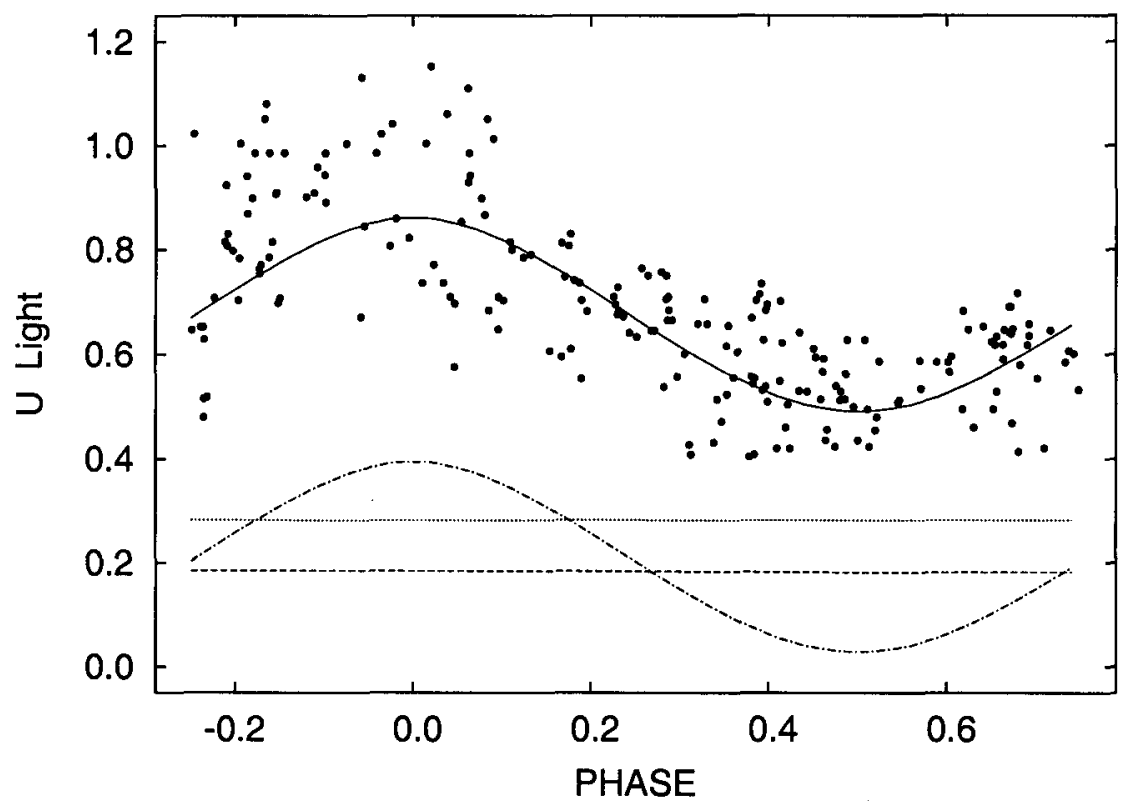

Figure 1. Separate chromospheric (dot-dash), wind (dashed) and star (dotted) light curves according to the circular orbit (simultaneous light/velocity) solution. The continuous line is the total light.

\section{A Pulsating Red Giant with Fluorescent Chromosphere}

Our Least-Squares solutions settled on a small eccentricity (approximately 0.04), but a fit with zero eccentricity fits about as well. Chromospheric fluorescence alone accounts for the $U$ variation, while $B$ variation is too small for wind and chromosphere to be distinguished and there is no perceptible wave in $V$. An earlier fluorescence model for AG Dra (Tomov, Tomova, \& Ivanova 2000) includes only wind emission, although they find strongest emission close to the red giant, so their result appears to be compatible with our all-chromosphere finding. Gális et al. (1999) identified a 355-day light curve and radial velocity period consistent with red star pulsation that we also find. That is, inclusion of a sine wave of adjustable period, phase, and amplitude improves the velocity fit significantly. 


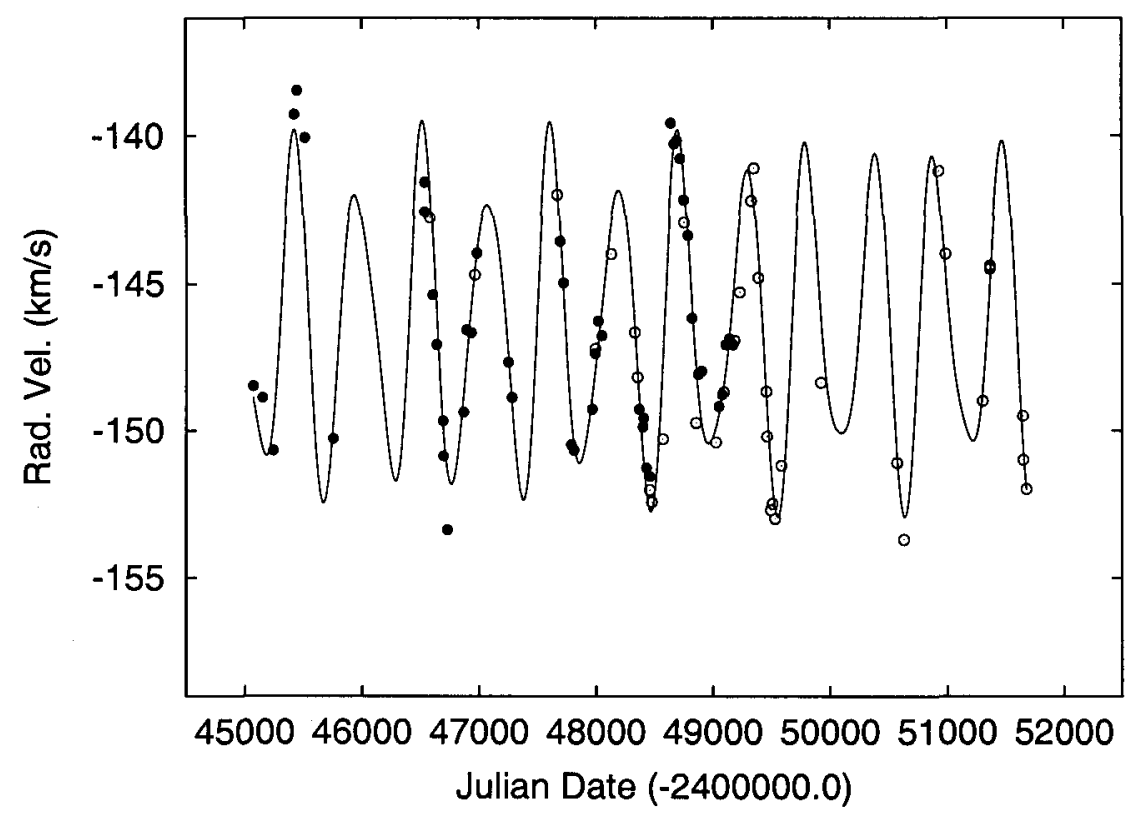

Figure 2. Fit to the KIII star velocities, including a pulsation sinusoid that reduces the standard error to $1.04 \mathrm{~km} \mathrm{~s}^{-1}$ from $1.47 \mathrm{~km} \mathrm{~s}^{-1}$ for a fit with no sinusoid. Filled circles are Mikołajewska et al. (1995), increased by $2.12 \mathrm{~km} \mathrm{~s}^{-1}$ (see Fekel et al. 2000); open circles are Smith et al. (1996) and Fekel et al. (2000).

\section{The Model as Scaffolding for Progress}

We believe that the wave mechanism in V1329 Cyg and AG Dra light curves is now understood in terms of a changing aspect of chromospheric and wind fluorescence. The binary fluorescence model gives reasonably definite results for the location and relative activity of those fluorescing regions. All of AG Dra's wave is found to come from its chromosphere, whereas V1329 Cyg's wave seems to have some contribution from the wind. Both symbiotics have far greater fluorescence in $U$ than in $B$ or $V$, but the contrast among photometric bands is much greater for AG Dra. Now one can save work on thermal re-radiation, which clearly is'a very minor effect in both binaries. It has also become clear that winds do not necessarily account for most fluorescence in symbiotic novae, and certainly not most of the fluorescent variation.

The next step will be to combine an optically thin radiative model with the physics and geometry of this model to synthesize spectra of local regions as they migrate in and out of view. The effort to do that can be justified only if spectra that are well distributed in time over full orbits can be observed. Meanwhile it may prove effective to apply the present model to other symbiotic stars. 
Table 1. Radial Velocity Solutions

\begin{tabular}{lccc}
\hline Parameter & $\begin{array}{c}\text { Circular } \\
d P / d t \text { fixed }\end{array}$ & $\begin{array}{c}\text { Non-circular } \\
d P / d t \text { fixed }\end{array}$ & $\begin{array}{c}\text { Non-circular } \\
d P / d t \text { adjusted }\end{array}$ \\
\hline$T_{0}$ (HJD) & $2448309.8 \pm 3.2$ & $2448309.6 \pm 3.2$ & $2448306.2 \pm 4.3$ \\
$P_{0}$ (days) & $549.02 \pm 0.87$ & $548.52 \pm 0.87$ & $548.32 \pm 0.89$ \\
$d P / d t$ & $-0.764410^{-3}$ & $-0.764410^{-3}$ & $-0.21 \pm 0.4710^{-3}$ \\
$e$ & 0.0 & $0.048 \pm 0.037$ & $0.041 \pm 0.037$ \\
$\omega$ (radians) & $\ldots$ & $5.70 \pm 0.67$ & $5.72 \pm 0.76$ \\
$V_{\gamma}\left(\mathrm{km} \mathrm{s}^{-1}\right)$ & $-146.43 \pm 0.16$ & $-146.42 \pm 0.16$ & $-146.40 \pm 0.15$ \\
$a\left(R_{\odot}\right)$ & $322 \pm 11$ & $325 \pm 12$ & $325 \pm 12$ \\
$i$ & 44.887 & 44.887 & 44.887 \\
$A_{\text {puls }}\left(\mathrm{km} \mathrm{s}^{-1}\right)$ & $1.41 \pm 0.17$ & $1.44 \pm 0.18$ & $1.40 \pm 0.17$ \\
$P_{\text {puls }}($ days) & $356.9 \pm 1.6$ & $356.8 \pm 1.6$ & $356.2 \pm 1.7$ \\
$T_{0 \text { puls }}(\mathrm{HJD})$ & $2448309.6 \pm 9.9$ & $2448310.4 \pm 9.6$ & $2448308.5 \pm 9.6$ \\
$\sigma\left(\mathrm{km} \mathrm{s}^{-1}\right)$ & 1.054 & 1.042 & 1.033 \\
\hline
\end{tabular}

\section{References}

Chochol, D., \& Wilson, R. E. 2001, MNRAS, 326, 437

Elias, N. M. II, Wilson, R. E., Olson, E. C., Aufdenberg, J. P., Guinan, E. F., Güdel, M., Van Hamme, W., \& Stevens, H. L. 1997, ApJ, 484, 394

Formiggini, L., \& Leibowitz, E. M. 1990, A\&A, 227, 121

Fekel, F. C., Hinkle, K. H., Joyce, R. R., \& Skrutskie, M. F. 2000, AJ, 120, 3255

Gális, R., Hric, L., Friedjung, M., \& Petrík, K. 1999, A\&A, 348, 533

Huang, C. C., Chen, Y. F., \& Chen, L. 1988, in The Symbiotic Phenomenon, ed. J. Mikołajewska, M. Friedjung, S. J. Kenyon, \& R. Viotti (Dordrecht: Kluwer), 205

Mikołajewska, J., Kenyon, S. J., Mikołajewski, M., Garcia, M. R., \& Polidan, R. S. 1995, AJ, 109, 1289

Munari, U., \& Zwitter, T. 2002, A\&A, 383, 188

Seaquist, E. R., Taylor, A. R., \& Button, S. 1984, ApJ, 284, 202

Seaquist, E. R., Taylor, A. R., \& Button, S. 1987, ApJ, 317, 555

Smith, V. V., Cunha, K., Jorissen, A., \& Boffin, H. M. J. 1996, A\&A, 315, 179

Tomov, N. A., Tomova, M. T., \& Ivanova, A. 2000, A\&A, 364, 557

Van Hamme, W., \& Wilson, R. E. 1998, BAAS, 30, 1402

Van Hamme, W., Samec, R. G., Gothard, N. W., Wilson, R. E., Faulkner, D. R., \& Branly, R. M. 2001, AJ, 122, 3436

Wilson, R. E. 1999, in Modern Astrometry and Astrodynamics, ed. R. Dvorak, H. F. Haupt, \& K. Wodnar (Vienna: Austrian Acad. Sci. Pub.), 219

Wilson, R. E. 2001, in Interacting Astronomers: A Symposium on Mirek Plavec's Favorite Stars, ed. P. Harmanec, P. Hadrava, \& I. Hubeny, Publ. Astr. Inst. Acad. Sci. Czech. No. 89, 60 
Table 2. Light and Velocity Solutions

\begin{tabular}{|c|c|c|c|}
\hline Parameter & Light Curves Only & $\begin{array}{c}\text { Simultaneous } \\
\text { Eccentric Orbit }\end{array}$ & $\begin{array}{l}\text { Simultaneous } \\
\text { Circular orbit }\end{array}$ \\
\hline$a\left(R_{\odot}\right)$ & 343.2011 & $326 \pm 55$ & $323 \pm 72$ \\
\hline$V_{\gamma}\left(\mathrm{km} \mathrm{s}^{-1}\right)$ & & $-146.420 \pm 0.047$ & $-146.430 \pm 0.046$ \\
\hline$T_{0}(\mathrm{HJD})$ & $2448293.4 \pm 5.1$ & $2448306.6 \pm 1.5$ & $2448306.8 \pm 1.4$ \\
\hline$P_{0}$ (days) & $548.3 \pm 1.1$ & $548.64 \pm 0.32$ & $548.83 \pm 0.32$ \\
\hline$d P / d t$ & $-0.88 \pm 0.4110^{-3}$ & $-0.76 \pm 0.2310^{-3}$ & $-0.77 \pm 0.2310^{-3}$ \\
\hline$e$ & $0.056 \pm 0.033$ & $0.042 \pm 0.014$ & 0.0 \\
\hline$\omega$ (radians) & $1.29 \pm 0.33$ & $5.71 \pm 0.28$ & \\
\hline$i$ & $44^{\circ} .9 \pm 6.6$ & $44.9 \pm 9.5$ & $45^{\circ} \pm 13^{\circ}$ \\
\hline$\Omega_{1}$ & $12.84 \pm 0.46$ & $12.0 \pm 1.5$ & $8.5 \pm 2.1$ \\
\hline$q=M_{2} / M_{1}$ & 0.33 & 0.33 & 0.33 \\
\hline$R_{1} / a$ & $0.0801 \pm 0.0029$ & $0.086 \pm 0.012$ & $0.123 \pm 0.032$ \\
\hline$R_{1} / R_{1 \text { lobe }}$ & 0.1757 & 0.1868 & 0.2571 \\
\hline$R_{1}\left(R_{\odot}\right)$ & $27.5 \pm 1.0$ & $28.1 \pm 6.0$ & $40 \pm 13$ \\
\hline$R_{2} / a$ & 0.000089 & 0.000099 & 0.00011 \\
\hline$T_{1}(\mathrm{~K})$ & 4300 & 4300 & 4300 \\
\hline$T_{\mathrm{wd}}(\mathrm{K})$ & 120,000 & 120,000 & 120,000 \\
\hline$d T_{\mathrm{wd}} / d t(\mathrm{~K} / \mathrm{d})$ & $-0.50 \pm 0.46$ & $-0.54 \pm 0.38$ & $-0.8 \pm 1.7$ \\
\hline$L_{1} / L_{2}(V)$ & $2046 \pm 677$ & $1904 \pm 475$ & $3188 \pm 1551$ \\
\hline$L_{1} / L_{2}(B)$ & $602 \pm 255$ & $560 \pm 186$ & $940 \pm 638$ \\
\hline$L_{1} / L_{2}(U)$ & $168 \pm 143$ & $156 \pm 125$ & $261 \pm 466$ \\
\hline$\epsilon_{\text {wind }}(V)$ & $0.14 \pm 0.18$ & $0.13 \pm 0.14$ & $0.13 \pm 0.39$ \\
\hline$\epsilon_{\text {wind }}(B)$ & $0.17 \pm 0.24$ & $0.16 \pm 0.18$ & $0.17 \pm 0.54$ \\
\hline$\epsilon_{\text {wind }}(U)$ & $0.10 \pm 0.20$ & $0.10 \pm 0.18$ & $0.13 \pm 0.59$ \\
\hline$\epsilon_{\text {chrom }}(V)$ & $0.003 \pm 0.060$ & $0.066 \pm 0.042$ & $0.036 \pm 0.026$ \\
\hline$\epsilon_{\text {chrom }}(B)$ & $0.064 \pm 0.052$ & $0.127 \pm 0.074$ & $0.078 \pm 0.062$ \\
\hline$\epsilon_{\text {chrom }}(U)$ & $0.56 \pm 0.46$ & $0.58 \pm 0.50$ & $0.44 \pm 0.81$ \\
\hline$\kappa_{\text {wind }}\left(\mathrm{cm}^{2} / \mathrm{g}\right)$ & 20.0 & 20.0 & 20.0 \\
\hline$\kappa_{\text {chrom }}\left(\mathrm{cm}^{2} / \mathrm{g}\right)$ & 20.0 & 20.0 & 20.0 \\
\hline$\rho_{\text {wind }}\left(\mathrm{g} \mathrm{cm}^{-3}\right)$ & $5.8810^{-16}$ & $5.8810^{-16}$ & $5.8810^{-16}$ \\
\hline$\rho_{\text {chrom }}\left(\mathrm{g} \mathrm{cm}^{-3}\right)$ & $6.0010^{-12}$ & $6.0010^{-12}$ & $6.0010^{-12}$ \\
\hline$H_{\text {pole }} / a$ & 0.005 & 0.005 & 0.005 \\
\hline$X_{\text {ionization }}$ & 5.00 & 5.00 & 5.00 \\
\hline$\lambda_{1}(\mathrm{~nm})$ & 5 & 5 & 5 \\
\hline$\lambda_{2}(\mathrm{~nm})$ & 350 & 350 & 350 \\
\hline$\lambda_{3}(V)(\mathrm{nm})$ & 501 & 501 & 501 \\
\hline$\lambda_{4}(V)(\mathrm{nm})$ & 580 & 580 & 580 \\
\hline$\lambda_{3}(B)(\mathrm{nm})$ & 389 & 389 & 389 \\
\hline$\lambda_{4}(B)(\mathrm{nm})$ & 484 & 484 & 484 \\
\hline$\lambda_{3}(U)(\mathrm{nm})$ & 340 & 340 & 340 \\
\hline$\lambda_{4}(U)(\mathrm{nm})$ & 393 & 393 & 393 \\
\hline$\lambda_{e f f}(V)(\mathrm{nm})$ & 540 & 540 & 540 \\
\hline$\lambda_{e f f}(B)(\mathrm{nm})$ & 437 & 437 & 437 \\
\hline$\lambda_{e f f}(U)(\mathrm{nm})$ & 366 & 366 & 366 \\
\hline
\end{tabular}

